\title{
Reanimación cardiopulmonar en pacientes con coronavirus SARS-CoV-2 en posiciones supino y prono
}

\section{Cardiopulmonary resuscitation in coronavirus SARS-CoV-2 patients in supine and prone positions}

Becket Argüello-López ${ }^{1}$ José Ricardo Navarro-Vargas²,3

\begin{abstract}
During this Pandemic it has been reported that $5 \%$ of cases require an Intensive Care Unit. Certain patients who present hypoxemia, acidosis, electrolyte disorders or adverse effects of drugs such as hydroxychloroquine, may end up in Cardiac Arrest. In these patients, it is recommended to provide high-quality cardiopulmonary resuscitation with all biosecurity measures. What should be done in the event of sudden cardiac arrest? The ethical obligation of health personnel is to resuscitate, but with the responsibility of personal protection, that is, with the precept of the rescuer's safety first. The purpose of this article is to review the protocol on the behavior recommended in the care of patients suffering from cardiac arrest in an environment with a COVID pandemic 19, such as that experienced by the world community today. For care and management protocol, AHA, UK and ILCOR guidelines, as well as publications on prone cardiopulmonary resuscitation, will be considered.
\end{abstract}

\section{RESUMEN}

Durante esta Pandemia se ha comunicado que un 5\% de los casos requieren de Unidad de Cuidados Intensivos. Determinados pacientes que presentan hipoxemia, acidosis, trastornos electrolíticos o efectos adversos de fármacos

\author{
Key words: \\ Resuscitation, \\ Pandemic, \\ SARS-COV-2; \\ Coronavirus, \\ personal protective \\ equipment, \\ cardiopulmonary \\ resuscitation, \\ prone position (MeSH)
}

\footnotetext{
Asociación Nicaragüense de Cuidados Intensivos. Managua, Nicaragua.

Universidad Nacional de Colombia, Facultad de Medicina, Decanatura. Bogotá D.C., Colombia.

Sociedad Colombiana de Anestesiología y Reanimación, Comité de Reanimación CLASA. Bogotá D.C., Colombia.
}

Fecha de recepción: 12 de agosto de 2020

Fecha de aceptación: 14 de agosto de 2020

\section{ORCID}

https://orcid.org/0000-0003-2548-1325

\section{Correspondencia:}

José Ricardo Navarro-Varga

Carrera 30 \# 45-03, Ciudad Universitaria, Universidad Nacional de Colombia, Facultad de Medicina, Decanatura.

Teléfono: 3165000

jrnavarrov@unal.edu.co 
como la hidroxicloroquina, pueden terminar en paro cardíaco. En estos pacientes se recomienda proporcionar una reanimación cardiopulmonar de alta calidad con todas las medidas de bioseguridad. ¿Qué se debe hacer frente al caso de un paro cardíaco súbito? La obligación ética del personal de salud es reanimar, pero con la responsabilidad de protección personal, es decir, con el precepto de primero la seguridad del reanimador. El propósito del presente artículo es revisar el protocolo sobre la conducta que se recomienda en la atención del paciente que sufre paro cardíaco en un ambiente con pandemia de COVID-19, como la que vive la comunidad mundial en la actualidad. Para la atención y protocolo de manejo, se tendrán en cuenta las guías de la AHA, del Reino Unido y de la ILCOR, así como las publicaciones de reanimación cardiopulmonar en posición prona.

\section{Palabras clave:}

Pandemias,

SARS-CoV-2,

coronavirus, equipo de protección

personal,

reanimación

cardiopulmonar, posición prona (DeCS)

\section{Introducción}

1 ada la actual pandemia por coronavirus, SARSCoV-2, frente a un paciente que presenta paro cardiaco se deberán tomar todas las precauciones del personal de salud antes de la reanimación cerebro-cardiopulmonar (RCP), pues si se toma en cuenta la experiencia en China, el $79 \%$ de los casos confirmados fueron contagiados por el $86 \%$ de pacientes que estaban asintomáticos[1].

Es importante considerar que los pacientes con SARS-CoV-2 y enfermedad cardiovascular tuvieron la tasa más alta de mortalidad (10,5\%). Las causas de estas complicaciones fueron las arritmias $(16,7 \%)$ y la lesión miocárdica aguda (7,2\%)[2].

El objetivo de este artículo de reflexión es resaltar las medidas pertinentes para la atención del paciente infectado con el coronavirus, SARS-CoV-2, en caso de precisar RCP, a partir de la revisión de las guías de la Asociación Americana del Corazón (American Heart Association, AHA)[3], del Reino Unido[4], y del Comité Internacional de Enlace en Reanimación (International Liaison Committee on Resuscitation, ILCOR) [5], como también las publicaciones sobre RCP en posición prona[6],[7], y la evidencia de su utilidad.

\section{Maniobras de reanimación}

Todo paciente (o representante legal en los casos de inconsciencia) que ingrese a la Unidad de Cuidados Intensivos deberá completar un consentimiento escrito de reanimar o verificar que tiene orden de no reanimación para que se pueda iniciar el algoritmo correspondiente según el ritmo de paro cardíaco.

El uso temprano de un desfibrilador aumenta significativamente las posibilidades de supervivencia y no aumenta el riesgo de infección[8].
Se debe seguir estrictamente la cadena de supervivencia, las Guías de la AHA[9], del Reino Unido[4], y del ILCOR[5], es decir, que una vez se haya diagnosticado inconsciencia en el paciente no intubado o con un ritmo de paro bajo cualquier modalidad de sedo-analgesia o relajación muscular, se debe activar inmediatamente el Código de Paro Cardiaco de la Unidad donde se encuentre la víctima. El equipo de reanimación avanzada deberá estar organizado desde el inicio de turno y con cada cambio de turno, y previamente se debe haber establecido el rol que va a desempeñar cada uno de sus integrantes para iniciar la reanimación.

Igualmente, todos los reanimadores deben disponer y haber hecho el entrenamiento previo de la utilización práctica de los equipos de protección personal (EPP) para atender a los pacientes infectados con coronavirus- SARS-CoV-2[10]. El número mínimo de reanimadores debe ser 3: un experto en la manipulación de la vía aérea, un integrante que realice las compresiones torácicas y otro que se encargue del monitor, del cardio-desfibrilador y de la administración de medicamentos. Cada 2 minutos se hará cambio entre los reanimadores que están a cargo de la vía aérea y las compresiones.

Las normas de bioseguridad exigen que quien realice las compresiones en un medio hospitalario debe tener un EPP[10]. La demanda física es bastante exigente, los trajes aumentan la temperatura corporal del reanimador, por lo que, puede experimentar visión borrosa y dificultad visual por el protector facial, lo cual puede interferir con una reanimación de calidad, y a causa de los efectos producidos por la fatiga se puede ver comprometida la calidad y eficacia de las compresiones[11] incluso, en escenarios de simulación se entorpece la intubación, el primer eslabón de las maniobras avanzadas de la reanimación (donde el 
acróstico ABCD secundario sigue vigente)[12]. Al respecto, se han publicado desde hace varios años, artículos donde se refleja la dificultad que ofrecen los EPP para realizar con eficacia diversas actividades[13],[14]. Sin embargo, el efecto de los talleres de reanimación con EPP puede modificar el ciclo de compresiones de 2 minutos a 1 minuto por reanimador para que éstas sean más efectivas[15].

De acuerdo a Tran et al.[16], los procedimientos de reanimación considerados de bajo riesgo para la generación de aerosoles y trasmisión de infección respiratoria aguda son la ventilación con máscara de oxígeno con filtro, las compresiones torácicas, la desfibrilación, la cardioversión, la aplicación de marcapaso, el acceso venoso e intraóseo; y los de alto riesgo son la ventilación con cánula nasal de alto flujo, la ventilación con ventilador manual, la ventilación mecánica no invasiva, la intubación traqueal y la broncoscopia.

Una de las maniobras más usadas en las unidades de cuidados intensivos (UCI) en los pacientes que presentan hipoxemia severa es ubicarlo en posición en prono; si el paciente presenta colapso circulatorio y cae en paro cardiaco en la posición en decúbito prono se deberá iniciar la RCP avanzada en esta posición. Existen publicaciones sobre reanimación cardiopulmonar en prono y directrices, como las del Consejo Europeo de Reanimación, en este grupo de pacien- tes[17],[18]. Cambiar la posición de un paciente grave de prono a supino es todo un desafío para el personal de salud altamente calificado. Al respecto, se recomienda iniciar la RCP en prono por los riesgos que se pueden presentar al momento de girarlo, como la extubación, perder líneas centrales y el tiempo que se tomaría en llevarlo a la posición supina[19].

Es mandatorio identificar y tratar las causas reversibles, a través del recurso de la nemotecnia de las $\mathrm{H}$ y las T del soporte vital cardiovascular avanzado[15]. Por ejemplo, si el paciente es tratado con hidroxicloroquina y azitromicina puede presentar paro cardiaco como efecto tóxico que conlleva a arritmias malignas tipo taquicardia ventricular, torsade de pointes o taquicardias ventriculares polimórficas[19],[20].

De acuerdo a las guías los fármacos de la reanimación (epinefrina, amiodarona) y la secuencia de las descargas no se modifican[3],[21].

Si el paciente con inestabilidad hemodinámica refiere haber estado en tratamiento con hidroxicloroquina y azitromicina se recomienda realizar electrocardiograma (ECG), gases arteriovenosos, electrolitos séricos y pruebas de función hepática y renal. Se recomienda aplicar la escala de riesgo de presentar QT prolongado (Tabla 1) [22].

Los pacientes hospitalizados que reciban estos fármacos deben tener un control estrecho de telemetría y monitoreo de $\mathrm{K}+$; ECG 2-3 horas después de

Tabla 1. Puntuación de riesgo para la prolongación QT corregido asociada a fármacos[22]

\begin{tabular}{lc}
\hline Factores de riesgos & Puntuación \\
Edad mayor a 68 años & 1 \\
Sexo femenino & 1 \\
Diuréticos de asa & 2 \\
K+ sérico menor a 3,5 meq/L & 2 \\
QTc mayor de 450 ms al ingreso & 2 \\
Infarto agudo de miocardio & 3 \\
2 o más fármacos que prolongan el QTC & 3 \\
Sepsis & 3 \\
Falla cardíaca & 3 \\
QTc prolongado por fármacos & 21 \\
Riesgo máximo por puntuación & Puntuación \\
Clasificación del riesgo & 6 o menos puntos \\
Riesgo bajo & 7 a 10 puntos \\
Riesgo moderado & 11 o más puntos \\
Riesgo alto & \\
\hline
\end{tabular}

QTc: Intervalo QT corregido; Fuente: Adaptado de 22. 
la segunda dosis de HCQ y posteriormente, cada 24 horas.

Se debe suspender la azitromicina y disminuir HCQ si: QTc aumenta + 60 ms o es $>500$ ms[23],[24].

\section{¿Qué contraindicaciones existen para las manio- bras de reanimación?}

Existen tres contraindicaciones ampliamente conocidas:

1. Dificultad del reanimador en soportar el EPP durante la reanimación.

2. Que el reanimador esté infectado con el COVID-19.

3. Que el reanimador tenga antecedentes de claustrofobia o golpe de calor[12].

\section{Manejo precoz de la vía aérea, una de las medi- das de control de la infección}

En el grupo de pacientes a intubar durante la fase de hipoxemia severa de acuerdo al CDC de Atlanta (11/03/2020) los procedimientos que generan aspiración de gotas en aerosol como la RCP, la intubación traqueal y la ventilación no invasiva entre otros, son de alto riesgo para la trasmisión de la infección viral[25]. Sólo cuando se disponga de todo el equipo requerido (ventilador mecánico, fuente de oxígeno, puerto de succión, equipo de vía aérea) y del personal capacitado con su EPP, se podrá iniciar el procedimiento. Estos lineamientos se aplican por igual al momento de iniciar la RCP avanzada en un paciente infectado con coronavirus SARS-CoV-2.

La reanimación se debe realizar en ambientes aislados y con el mínimo personal necesario y donde se disponga de manera apropiada de todos los elementos de bioseguridad. Cuando se requiere la intubación traqueal se debe hacer de manera precoz por un experto (anestesiólogo/intensivista/especialista en medicina de urgencias).

Hay que evitar la diseminación del virus por movilización de gotas en aerosol de la víctima (ventilación con ventilador manual, con presión positiva, nebulizadores). Para el transporte del paciente infectado que es reanimado, se deben tomar todas las precauciones para evitar el contagio, además, de las medidas de protección personal, debe ir el menor número de reanimadores posible[25]. El algoritmo del manejo de la RCP en el paciente con COVID-19 se representa en la Figura 1.

Si el paciente está intubado en posición prono y hace paro cardíaco, se pueden realizar compresiones en el dorso, entre las escápulas, con una profundidad de 6 centímetros a un ritmo de 120 compresiones por minuto[18],[26].

La reanimación cardiopulmonar en prono (RCPP) fue propuesta por primera vez por McNeil en 1989[27]. Estudios realizados por Brown et al.[28], Mazer et al. (2003)[29] y Wei et al. (2006)[30] han encontrado resultados favorables con la RCP en posición en prono (Figura 2).

Las pautas del Consejo de Reanimación del Reino Unido[31], publicadas en 2014, recomiendan que las compresiones torácicas se inicien sin ningún cambio de posición en pacientes adultos que presentan un paro cardíaco durante procedimientos de neurocirugía. La eficacia de las compresiones debe ser evaluada con la capnografía y la presión arterial; si las compresiones no son efectivas se debe poner al paciente en posición supina. Las pautas de la AHA[3],[32] para la RCP y la atención cardiovascular de emergencia recomiendan que cuando el paciente no puede colocarse en posición supina, puede ser razonable que los reanimadores proporcionen $\mathrm{RCP}$ con el paciente en posición prono, particularmente en pacientes hospitalizados con la vía aérea asegurada[32].

Desde antes de las guías AHA 2000, Sun et al.[33] ya habían reportado 2 reanimaciones exitosas en pacientes neuroquirúrgicos que presentaron paro cardiaco en posición prona. Las compresiones torácicas se realizaron sobre la columna torácica media y otro reanimador colocaba su mano en la parte inferior del esternón como contrapresión (compresión precordial inversa).

La literatura revisada[26],[31] considera que hay pocos estudios sobre las maniobras de RCP en posición prona y recomiendan una técnica "a 2 manos", un reanimador coloca sus manos sobre la columna torácica media, entre las escápulas, y otro reanimador hace contrapresión con una mano debajo del tercio inferior del esternón de la víctima (Figura 3). Para lograr una desfibrilación exitosa, los parches o las paletas del desfibrilador se deben colocar, uno en la línea axilar media del lado izquierdo y el otro sobre la escápula derecha, o en las regiones biaxilares[26] (Figura 4).

\section{¿Cuándo no iniciar la reanimación?}

Se debe establecer una balanza entre los recursos institucionales y las expectativas de vida del paciente dentro de un marco ético. La humanización debe ser inherente al ejercicio profesional[34] y aplicar en cada conducta médica la mente respetuosa y la mente ética; por esta razón, en estas decisiones, debe primar la concepción del ser humano en toda su integridad y 


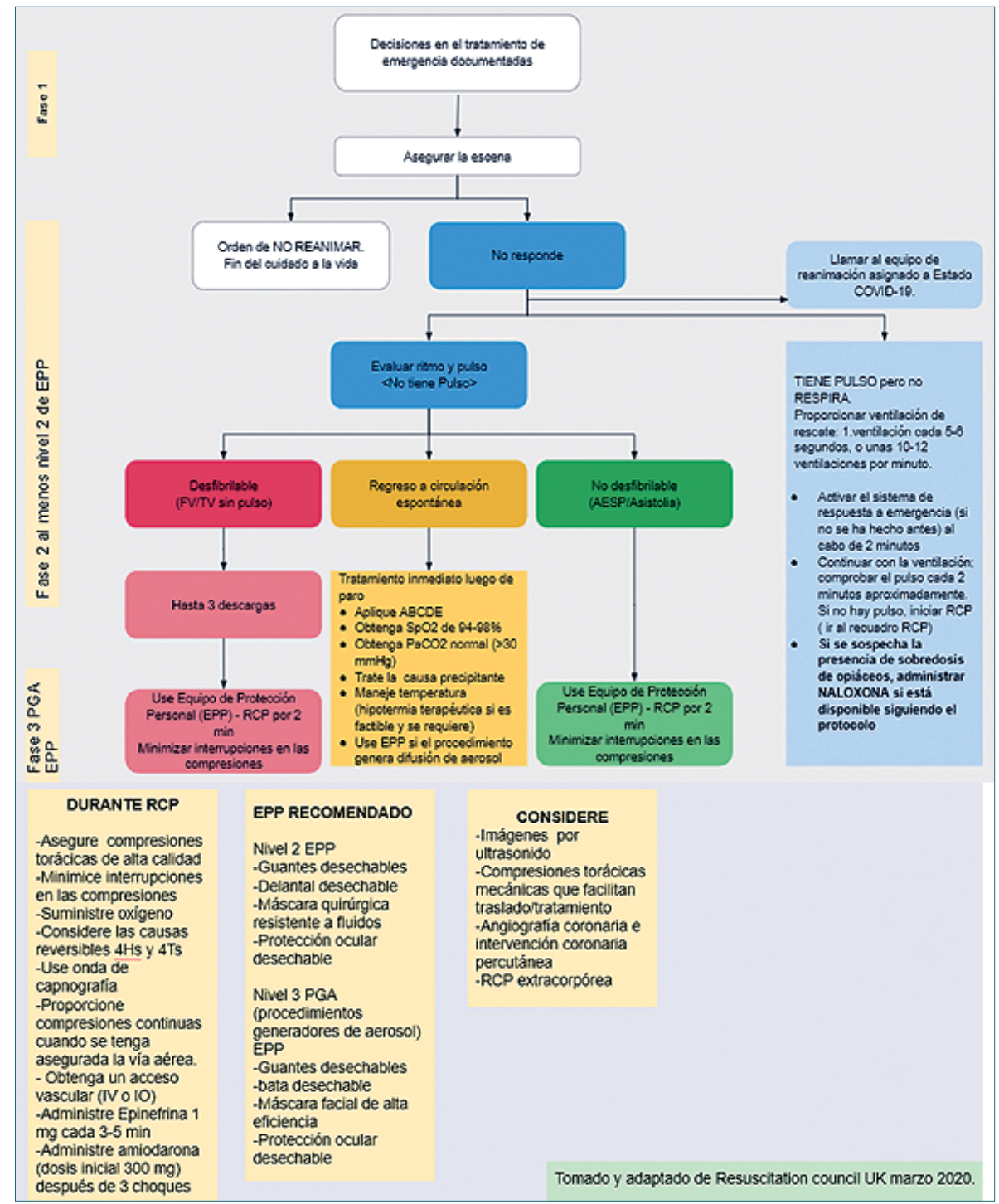

Figura 1. Soporte vital avanzado para pacientes con COVID-19. Fuente: Tomado y adaptado de 4. no prolongar su agonía a toda costa.

Hay pacientes cuyo pronóstico es pésimo y, además, se cuenta con deficiente número de camas en UCI. Condiciones clínicas irreversibles como hipoxemia refractaria y falla multiorgánica con una puntuación SOFA que haya empeorado mínimo 2 puntos en las últimas 48 horas; pacientes mayores de 80 años con multimorbilidad (falla cardiaca, falla renal crónica, EPOC, cirrosis, carcinoma en fase terminal, $\mathrm{VIH}$ muy comprometido, etc.); pacientes con enfermedad mental severa o enfermedades degenerativas en sepsis severa, etc.[35].

\section{Conclusión}

La RCP es un procedimiento eficaz, viable e importante en un paciente que ha sido víctima de paro cardiaco, así sea sospechoso o sufra una infección de gran contagio como el COVID-19. Es la responsabilidad ética del personal de salud, poder brindarle la oportunidad de reanimación a la víctima, como también asumir este reto con todas las medidas de bioseguridad y protección personal.

Es importante durante la RCP en posición supino o prono, después de haber iniciado las maniobras, 


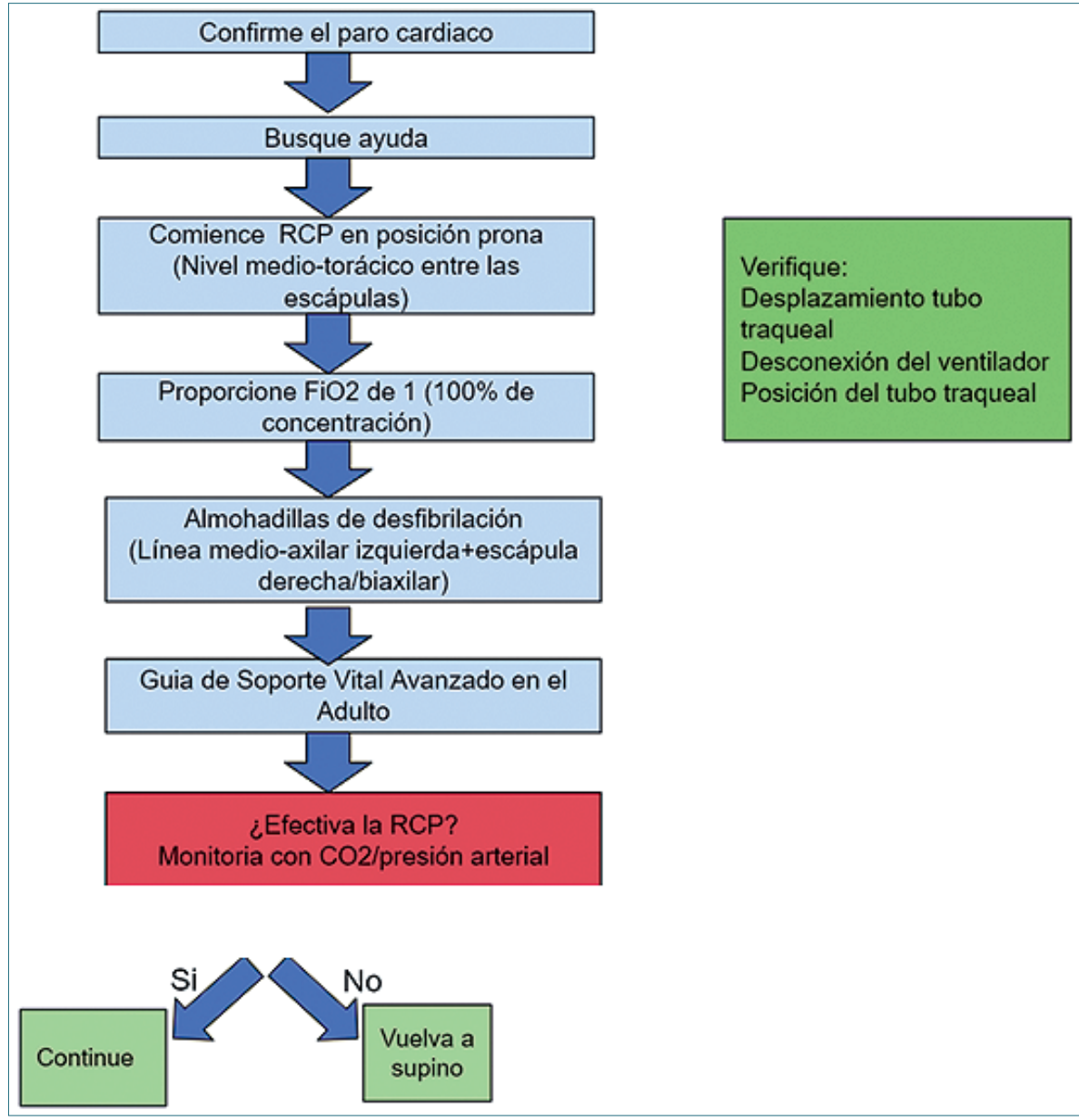

Figura 2. Reanimación cardiopulmonar en posición prina (P-RCP). Fuente: tomado y adaptado de 26.

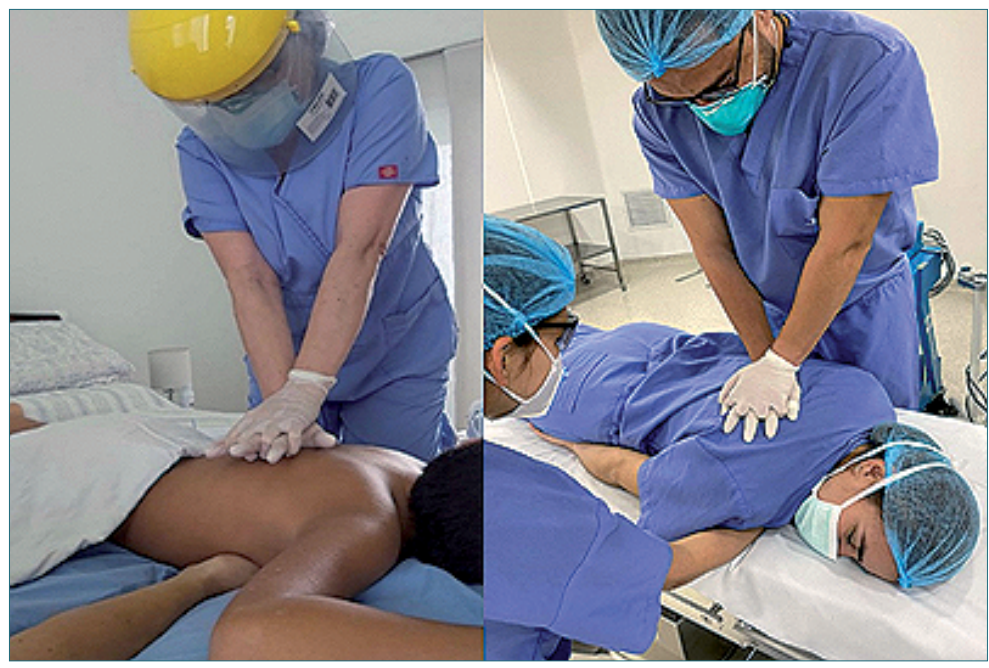

Figura 3. Posición prona y $\mathrm{RCP}$ con 2 reanimadores (observe la mano del $2^{\circ}$. reanimador debajo del tercio inferior del esternón). 


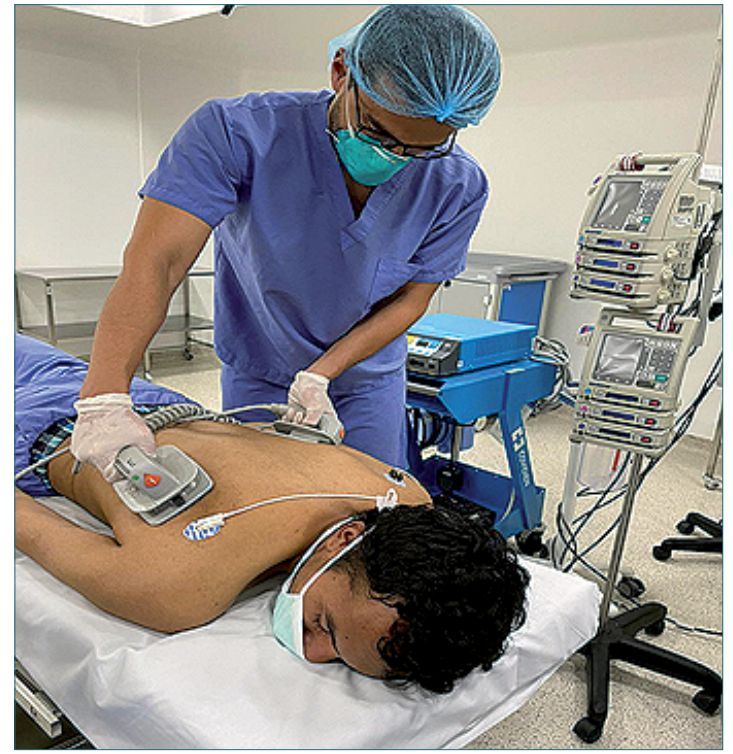

Figura 4. Desfibrilación en posición prona. evaluar el ritmo cardiaco con ECG y el gasto cardiaco de manera indirecta, a través de la capnografía. Para descontinuar o suspender las maniobras se deben aplicar los lineamientos actuales de RCP (ILCOR/AHA/ Reino Unido).

\section{Conflicto de intereses}

Ninguno reportado por los autores.

\section{Financiación}

Ninguna reportada por los autores.

\section{Agradecimientos}

A las doctoras Lucía Pimiento y Rayssa Minella Becerra por facilitar las fotos de las Figuras 3 y 4 . Y a Camila Navarro por la elaboración de las Figuras 1 y 2.

\section{Referencias}

1. Huang $C$, Wang $Y$, Li $X$, Ren L, Zhao J, Hu Y, Zhang L, Fan G, Xu J, Gu X, Cheng Z, Yu T, Xia J, Wei $Y$, Wu W, Xie $X$, Yin W, Li H, Liu M, Xiao Y, Gao H, Guo L, Xie J, Wang G, Jiang R, Gao Z, Jin Q, Wang J, Cao $B$. Clinical features of patients infected with 2019 novel coronavirus in Wuhan, China. Lancet. 2020; 395 (10223): 497-506. https://doi.org/10.1016/S01406736(20)30183-5

2. Anderson RM, Heesterbeek $H_{\text {, }}$ Klinkenberg D, Hollingsworth T. How will country-based mitigation measures influence the course of the COVID-19 epidemic?. Lancet. 2020; 395 (10228): 931-934. https:// doi.org/10.1016/S01406736(20)30567-5

3. Interim Guidance for Basic and Advanced Life Support in Adults, Children, and Neonates With Suspected or Confirmed
COVID-19: From the Emergency Cardiovascular Care Committee and Get With the Guidelines ${ }^{{ }_{-}}$Resuscitation Adult and Pediatric Task Forces of the American Heart Association in Collaboration with the American Academy of Pediatrics, American Association for Respiratory Care, American College of Emergency Physicians, The Society of Critical Care Anesthesiologists, and American Society of Anesthesiologists: Supporting Organizations: American Association of Critical Care Nurses and National EMS Physicians. Edelson D, Sasson C, Chan PS, Atkins DL, Aziz K, Becker $L$, et al. Circulation AHA 2020. https://www.ahajournals. org/doi/pdf/10.1161/CIRCULATIONAHA.120.047463

4. Guidance for the resuscitation of adult COVID-19 patients in acute hospital settings. https://www. resus.org.uk/media/statements/ resuscitation-council-uk-statements-on-covid-19-coronavirus- cpr-and-resuscitation/covidhealthcare-resources

5. Couper K, Taylor-Phillips S, Grove A, Freeman K, Osokogu O, Court R, et al. COVID-19 infection risk to rescuers from patients in cardiac arrest. Consensus on Science with treatment recommendations (internet) Brussels, Belgium; International Liaison Committee on Resuscitation (ILCOR), 2020 https://costr.ilcor.org/document/ covid-19-infection-risk-to-rescuers-from-patients-in-cardiac-arrest

6. Dequin P-F, Hazouard E, Legras A, Lanotte R, Perrotin D. Cardiopulmonary resuscitation in the prone position: kouwenhoven revisited. Intensive Care Med 1996; 22:1272. https://doi. org/10.1007/BF01709349

7. Gomes D de S, Bersot CDA. Cardiopulmonary resuscitation in the prone position. Open J Anesthesiol 2012; 2: 19920. https://doi.org/10.4236/ 
ojanes.2012.25045

8. Covid-19. Practical Guidance for Implementation. https://www. ilcor.org/covid-19, revisado Julio 2 de 2020

9. Meaney PA, Bobrow BJ, Mancini ME, Christenson J, de Caen AR, Bhanji F, Abella BS, Kleinman ME, Edelson DP, Berg RA, Aufderheide TP, Menon V, Leary M; en representación de los investigadores de la CPR Quality Summit, el Emergency Cardiovascular Care Committee de la American Hearth Association y el Council on Cardiopulmonary, Critical Care, Perioperative and Resuscitation. Calidad de la reanimación cardiopulmonar: mejora de los resultados de la reanimación cardíaca intra y extrahospitalaria. Declaración de consenso de la American Hearth Association. Circulation. 2013; 128:417435. https://doi.org/10.1161/ CIR.0b013e31829d8654

10. Requerimientos para uso de equipos de protección personal (EPP) para el nuevo coronavirus (2019-nCoV) en establecimientos de salud disponible en https:// www.paho.org/hq/index. php?option=com_content\&vie $w=$ article\&id=15720:technicaldocuments-coronavirus-diseasecovid-19\&ltemid=4206\&lang=es, revisado junio 27 de 2020.

11. Chen J, Lu KZ, Yi B, Chen Y. Chest Compression With Personal Protective Equipment During Cardiopulmonary Resuscitation A Randomized Crossover Simulation Study. Medicine. 2016; 95 (14). https://doi.org/10.1097/ MD.0000000000003262

12. Greenland KB, Tsui D, Goodyear $\mathrm{P}$, et al. Personal protection equipment for biological hazards: does it affect tracheal intubation performance. Resuscitation. 2007;74:119-126 https:// doi.org/10.1016/j.resuscitation.2006.11.011
13. Coates MJ, Jundi AS, James MR. Chemical protective clothing: a study into the ability of staff to perform lifesaving procedures. J Accid Emerg Med. 2000; 17: 115-118. https://doi. org/10.1136/emj.17.2.115

14. Kim TH, Kim Ch H, Shin SD, Haam S. Influence of personal protective equipment on the performance of life-saving interventions by emergency medical service personnel. Simulation: Transaction of the Society for Modeling and Simulation International. 2016 92 (10): 893-898. https://doi. rg/10.1177/0037549716662322

15. Malysz M, Dabrowski M, Bottiger BW, Smereka J, Kulak K, et al. Resuscitation of the patient with suspected/confirmed COVID-19 when wearing personal protective equipment: A randomized multicenter crossover simulation trial. Cardiol J 2020. https://www.researchgate.net/ publication/341398321_Resuscitation_of_the_patient_with_suspectedconfirmed_COVID-19_ when_wearing_personal_protective_equipment_A_randomized_multicenter_crossover_simulation_trial revisado julio 2 de 2020. https://doi.org/10.5603/ CJ.a2020.0068

16. Tran K, Cimon K, Severn $M$, Pessoa-Silva CL, Conly J. Aerosol Generating Procedures and Risk of Transmission of Acute Respiratory Infections to Healthcare Workers: A Systematic Review. PLoS ONE. 2012; 7(4): e35797. https://doi.org/10.1371/journal. pone.0035797

17. Wei J, Tung D, Sue SH, Wu SV, Chuang Y Ch. Cardiopulmonary Resuscitation in Prone Position: A Simplified Method for Outpatients. J Clin Med Assocd 2006; 69 (5): 202-206 https://doi.org/10.1016/\$17264901(09)70219-9
18. European Resuscitation Council COVID-19 Guidelines. https://erc.edu/ s/5714e77d5e615861f00f7d18/ content_entry5ea884fa4c84867 335e4d1ff/5ea885f34c8486733 5e4d20e/files/ERC_covid19_pages.pdf?1588257310, revisado junio 29 de 2020.

19. Guidance For: Prone Positioning in Adult Critical Care Churchill House Red Lion Square London. https://www.ficm.ac.uk/sites/ default/files/prone_position_in_ adult_critical_care_2019.pdf

20. Moitra VK, Gabrielli A, Maccioli GA, O'Connor MF. Anesthesia advanced circulatory lifesupport. Can J Anesth. 2012; 59(6):586$60 \mathrm{https}: / /$ doi.org/10.1007/ s12630-012-9699-3

21. ACLS Cardiac Arrest Algorithm for Suspected or Confirmed COVID-19 Patients https://cpr. heart. org/-/media/cpr-files/resources/ covid-19-resources-for-cpr-.training/english/algorithmacls_cacovid_200406.pdf?la=en revisado Julio 1 de 2020

22. Tisdale JE, Jaynes HA, Kingery JR, Mourad NA, Trujillo TN, Overholser BR, et al. Development and validation of a risk score to predict QT interval prolongation in hospitalized patients. Circulation 2013; 6(4): 479-487 https:// doi.org/10.1161/CIRCOUTCOMES.113.000152

23. Simpson T, Salazar J, Vittinghoff $\mathrm{E}$, et al. Association of QT prolonging medications with risk of autopsy causes of sudden death. JAMA Int Med. 2020;180(5):1-9. https://doi.org/10.1001/jamainternmed.2020.0148

24. Giudicessi JR, Noseworthy PA, Friedman PA, Ackerman MJ. Urgent Guidance for Navigating and Circumventing the QTC Prolonging and Torsadogenic Potential of Possible Pharmacotherapies for COVID-19. Mayo Foundation 
for Medical Education and Research. Mayo Clin Proc. 2020;95 https://doi.org/10.1016/j. mayocp.2020.03.024

25. Interim Guidance for Healthcare Providers during COVID-19 outbreak. American Heart Association 2020. https://www.heart. org/en/about-us/coronaviruscovid-19-resources

26. Bamford P, Denmade C, Newmarch C, Shirley $P$, singer B, Webb $S$, et al. Guidance For: Prone Positioning in Adult Critical Care. Intensive Care Society and Faculty of Intensive Care medicine. 2019. https://www. ficm.ac.uk/sites/default/files/ prone_position_in_adult_critical_care_2019.pdf

27. McNeil EL. Re-evaluation of cardiopulmonary resuscitation. Resuscitation 1989;18: 1-5. https://doi.org/10.1016/03009572(89)90107-X

28. Brown J, Roger J, Soar J. Cardiac arrest during surgery and ventilation in the prone position: a case report and systematic review. Resuscitation 2001;50: 233-8. https://doi.org/10.1016/\$03009572(01)00362-8

29. Mazer SP, Weisfeldt M, Bai D, Cardinale C, Arora R, Ma C, Sciacca RR, et al. Reverse CPR: a pilot study of CPR in the prone position. Resuscitation 2003; 57: 279-85. https:// doi.org/10.1016/S03009572(03)00037-6

30. Wei J, Tung $D$, Sue SH, Wu SV, Chuang YC, Chang CY. Cardiopulmonary resuscitation in prone position: A simplified method for outpatients. J Chin Med Assoc 2006; 69: 202-6. https://doi.org/10.1016/\$17264901(09)70219-9

31. Resuscitation Council (UK). Management of cardiac arrest during neurosurgery in adults. Guidelines for healthcare providers. 2014. https://www.resus.org.uk/ publications/management-ofcardiac-arrest-during-neurosurgery-in-adults/
32. 2015 American Heart Association guidelines update for Cardiopulmonary Resuscitation and emergency cardiovascular care. Circulation 2015; 132:18: Supplement 2.

33. Sun W, Huang F, Kung $K$, Fan $\mathrm{S}$, Chen T. Successful Cardiopulmonary resuscitation of two patients in the prone position using reversed precordial compression. Anesthesiology 1992;77(1):202-204. https:// doi.org/10.1097/00000542199207000-00027

34. Navarro-Vargas JR. Humanización en salud. Editorial. Rev Fac Med. 2018; 66(3): 291-292 https://doi.org/10.15446/revfacmed.v66n3.75011

35. Ethical Framework for Health Care Institutions Responding to Novel Coronavirus SARS-CoV-2 (COVID-19); Guidelines for Institutional Ethics Services Responding to COVID-19: https://www. thehastingscenter.org/ethicalframeworkcovid19 